\title{
Towards Understanding Tribocorrosion in Hip Replacements
}

\author{
Emily Hoffman, Yifeng Liao, and Laurence D. Marks
}

Department of Materials Science and Engineering, Northwestern University, Evanston, IL

Total hip replacement is one of the most successful treatments for patients with end-stage disease of the hip, and by 2030 the number is expected to reach 572,000 annually. As patients live longer, the excellent mechanical properties of metal-on-metal (MoM) implants could hold promise as an alternative to ultrahigh-molecular-weight polyethylene (MoP) traditional implants [1]. In addition, recent data suggests that tribocorrosion at the taper joints of hip replacements components could be a major factor in hip health issues.

Preferentially corroded CoCrMo grain boundaries were examined through transmission electron microscopy (TEM) to investigate the boundaries in retrieved hip implants The crystallographic structure was studied to determine the grain boundaries orientation, and the chemical composition was studied to quantify segregation at the grain boundaries. Initial results of local energy-dispersive x-ray spectroscopy (EDS) show the enhancement of $\mathrm{Cr}$ and depletion or $\mathrm{Co}$ at high-energy grain boundaries.

The misorientation of the grain boundaries was determined through electron backscattered diffraction (EBDS) and was quantified using coincident site lattice geometries $(\Sigma 3-\Sigma 35)$ using the tolerance as defined by the Brandon Criterion, $\Delta \theta \leq 15^{\circ}\left(\Sigma^{-1 / 2}\right)$ [2]. An SEM in Figure 1 (A) shows the visual difference between corroded and immune grain boundaries, while (B) shows the EBSD mapped crystallographic orientations. Previous work on the crystallographic misorientation for 102 corroded and immune grain boundaries showed that 100\% low-energy grain boundaries did not corrode, whereas only $30.4 \%$ of general high-angle grain boundaries did not corrode [3]. This indicates that the crystallographic orientation affects corrosion susceptibility in CoCrMo. Using this knowledge of grain boundary orientation, focused ion beam (FIB) was used to make TEM samples of specifically selected, high-energy grain boundaries to evaluate the composition across the boundary.

EDS was employed to scan line profiles across the high-energy grain boundaries of the CoCrMo TEM samples. Figure 2 (A) and (B) show an example TEM sample FIB' ed from a corroded grain boundary. Figure 2 (C) shows the EDS scan area, with (D) depicting the alloy element compositions across the scan. EDS results indicate that, at the corroded boundary, there is a depletion of cobalt and an enrichment of both carbon and chromium around approximately $10 \mathrm{~nm}$ on either side of the boundary. This trend of Co depletion and $\mathrm{Cr}$ enhancement is seen in three out of four samples initially evaluated, to various degrees. Our preliminary results showed composition along the depth of the grain boundary changes, necessitating further TEM analysis to determine the segregation levels and atomic structure of corrosion susceptible grain boundaries.

Grain boundaries are known to be an initiation point for corrosion. Standard engineering methods show that a sacrificial anode corrodes faster, yet protects the remainder of the material. The role of this localized corrosion is uncertain. Using EDS, it has been shown that in addition to crystallographic orientation, chemical segregation is also present at corroded CoCrMo grain boundaries. 


\section{References:}

[1] Health at a Glance: Europe 2010. Oecd, (2010).

[2] Randle, V. and O. Engler, Introduction to texture analysis. 2000: CRC Press.

[3] Panigrahi, P., et al, In Preparation, (2012).

[4] The authors acknowledge funding from the NSF on grant number CMMI-1030703 and by the NIH on grant number 1RC2AR058993-01.

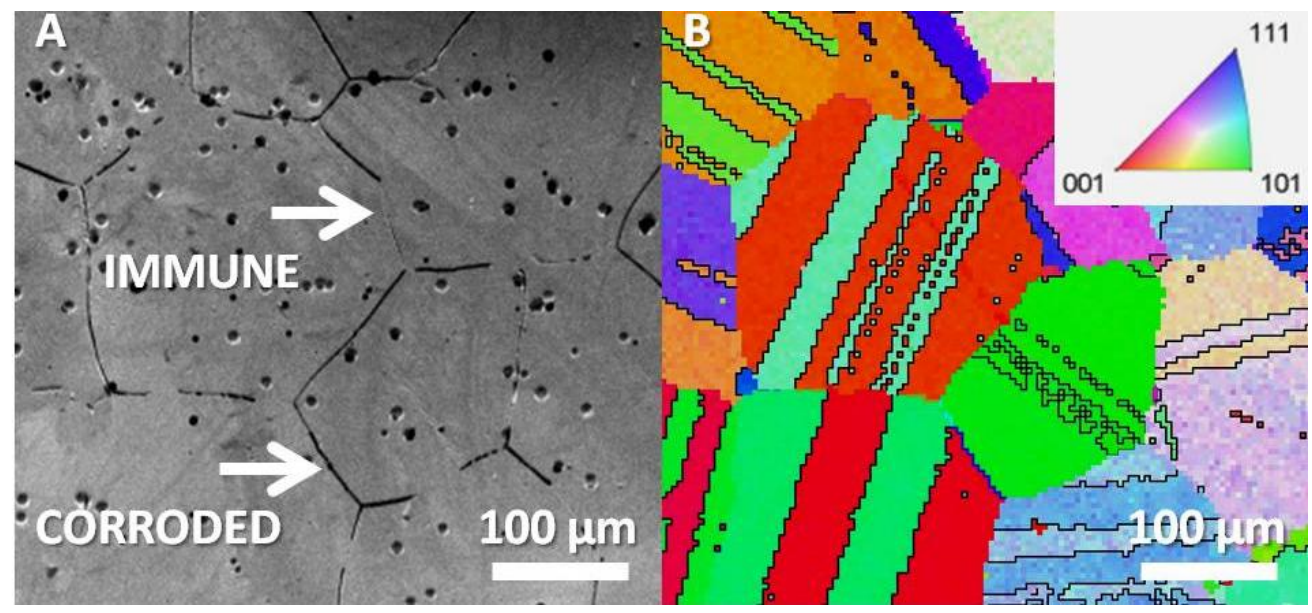

Figure 1. (A) A SEM image showing the corroded and immune grain boundaries in a CoCrMo sample, along with the EBSD crystallographic mapping to determine the grain boundary energy in (B).

Figure 2. (A) FIB/SEM image showing a single grain boundary being cut from the sample, viewed in a TEM in (B) and (C) and EDS spectra indicating Co depletion (purple) and $\mathrm{Cr}$ increase (red) near the boundary marked with the dashed line in (D).
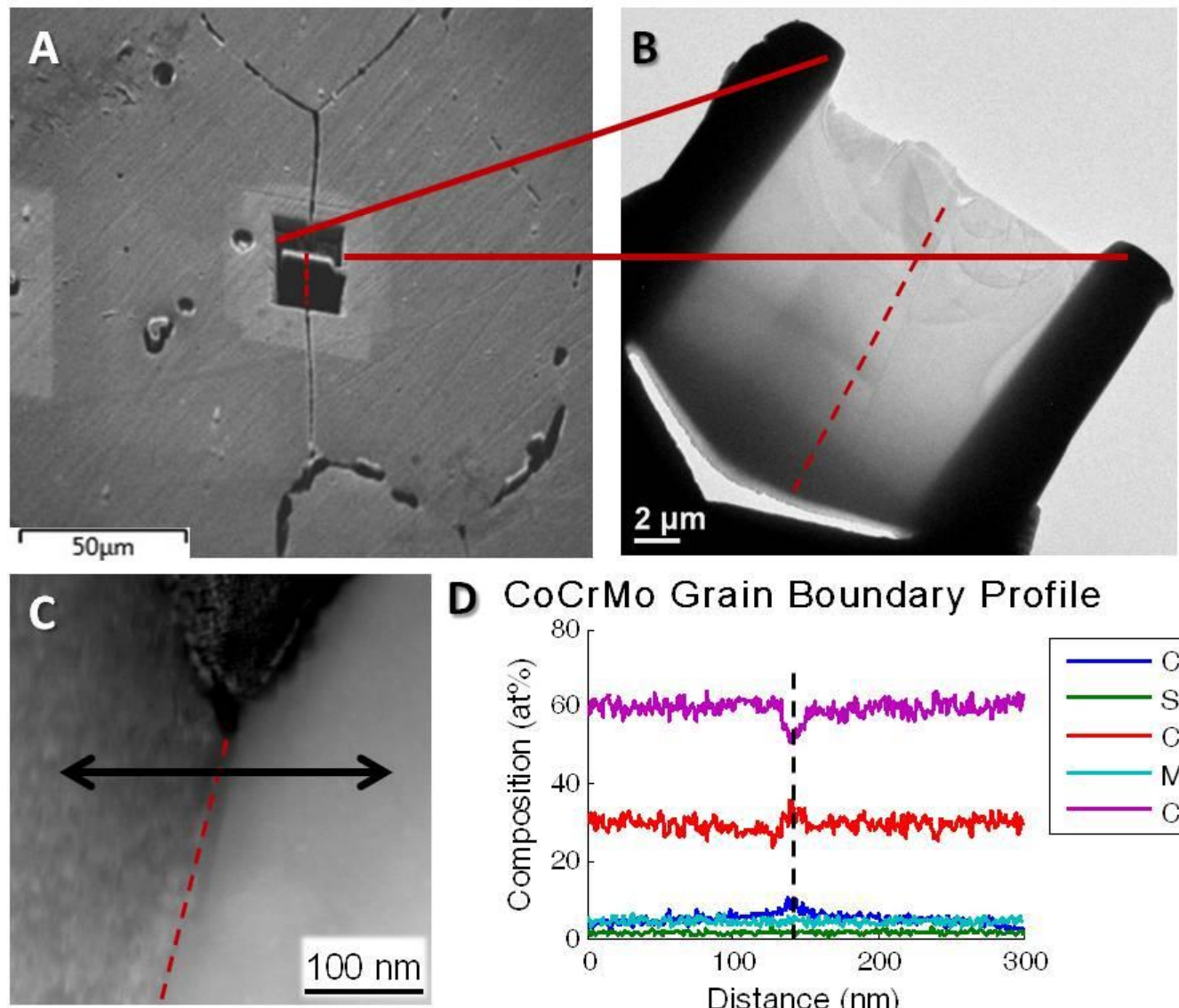

D CoCrMo Grain Boundary Profile

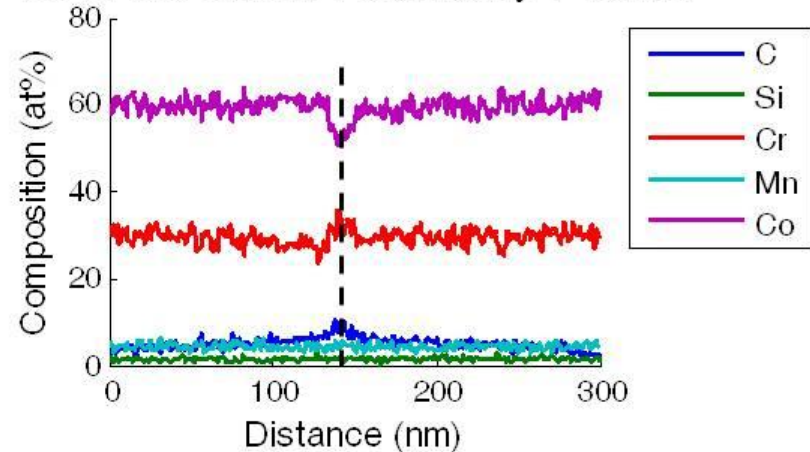

